This item was submitted to Loughborough's Research Repository by the author.

Items in Figshare are protected by copyright, with all rights reserved, unless otherwise indicated.

\title{
The effect of physical fatigue on oscillatory dynamics of the sensorimotor cortex
}

PLEASE CITE THE PUBLISHED VERSION

http://dx.doi.org/10.1111/apha.12843

\section{PUBLISHER}

(c) 2016 Scandinavian Physiological Society. Published by John Wiley \& Sons Ltd

VERSION

AM (Accepted Manuscript)

\section{PUBLISHER STATEMENT}

This work is made available according to the conditions of the Creative Commons Attribution-NonCommercialNoDerivatives 4.0 International (CC BY-NC-ND 4.0) licence. Full details of this licence are available at: https://creativecommons.org/licenses/by-nc-nd/4.0/

\section{LICENCE}

CC BY-NC-ND 4.0

\section{REPOSITORY RECORD}

Fry, Adam, Karen J. Mullinger, George C. O'Neill, Matthew J. Brookes, and Jonathan P. Folland. 2019. "The Effect of Physical Fatigue on Oscillatory Dynamics of the Sensorimotor Cortex". figshare. https://hdl.handle.net/2134/24406. 


\title{
The effect of physical fatigue on oscillatory dynamics of the sensorimotor cortex
}

\author{
Adam Fry ${ }^{1}$, Karen J. Mullinger ${ }^{2,3}$, George C. O’Neill ${ }^{2}$, Matthew J. Brookes ${ }^{2}$ and \\ Jonathan P. Folland ${ }^{1}$
}

${ }^{1}$ School of Sport, Exercise and Health Sciences, Loughborough University, Leicestershire, LE11 3TU, United Kingdom

${ }^{2}$ Sir Peter Mansfield Imaging Centre, School of Physics and Astronomy, University of Nottingham, University Park, Nottingham, NG7 2RD, United Kingdom

${ }^{3}$ Birmingham University Imaging Centre, School of Psychology, University of Birmingham, Birmingham, B15 2TT, United Kingdom

*Correspondence to:

Adam Fry,

School of Sport Exercise \& Health Sciences,

Loughborough University,

Leicestershire,

LE113TU

UK

E-mail: a.fry3@lboro.ac.uk

Short Title: Sensorimotor oscillations during fatigue 


\section{ABSTRACT}

Aim: While physical fatigue is known to arise in part from supraspinal mechanisms within the brain exactly how brain activity is modulated during fatigue is not well understood. Therefore, this study examined how typical neural oscillatory responses to voluntary muscle contractions were affected by fatigue.

Methods: Eleven healthy adults (age $27 \pm 4$ years) completed two experimental sessions in a randomised crossover design. Both sessions first assessed baseline maximal voluntary isometric wrist-flexion force $\left(\mathrm{MVF}_{\mathrm{b}}\right)$. Participants then performed an identical series of fourteen test contractions $\left(2 \times 100 \% \mathrm{MVF}_{\mathrm{b}}, 10 \times 40 \% \mathrm{MVF}_{\mathrm{b}}, 2 \times\right.$ $100 \% \mathrm{MVF}_{\mathrm{b}}$ ) both before and after one of two interventions: forty 12 -s contractions at $55 \% \mathrm{MVF}_{\mathrm{b}}$ (fatigue intervention) or $5 \% \mathrm{MVF}_{\mathrm{b}}$ (control intervention). Magnetoencephalography (MEG) was used to characterise both the movement-related mu and beta decrease (MRMD and MRBD) and the post-movement beta rebound (PMBR) within the contralateral sensorimotor cortex during the $40 \% \mathrm{MVF}_{\mathrm{b}}$ test contractions, while the $100 \% \mathrm{MVF}_{\mathrm{b}}$ test contractions were used to monitor physical fatigue.

Results: The fatigue intervention induced a substantial physical fatigue that endured throughout the post-intervention measurements $(28.9-29.5 \%$ decrease in MVF, $\mathrm{P}<0.001$ ). Fatigue had a significant effect on both PMBR (ANOVA, session $\times$ timepoint interaction: $\mathrm{P}=0.018)$ and $\mathrm{MRBD}(\mathrm{P}=0.021)$ : the magnitude of $\mathrm{PMBR}$ increased following the fatigue but not the control interventions, whereas MRBD was decreased post-control but not post-fatigue. $\mathrm{Mu}$ oscillations were unchanged throughout both sessions. 
Conclusion: Physical fatigue resulted in an increased PMBR, and offset attenuations in MRBD associated with task habituation.

Keywords: magnetoencephalography; MEG; motor; sensory; mu; beta; event-related desynchronization; event-related synchronization 


\section{INTRODUCTION}

Physical fatigue can be defined as a reversible decline in the force generating capacity of the neuromuscular system. During physical activity, fatigue arises not only from peripheral processes within the active skeletal muscle(s) but also from supraspinal mechanisms within the brain (Gandevia 2001). In fact, studies utilising transcranial magnetic stimulation (TMS) have demonstrated supraspinal fatigue (a component of central fatigue relating to a progressively suboptimal output from the motor cortex) can account for as much as $66 \%$ of the exhibited physical fatigue during a prolonged lowintensity muscle contraction (Smith et al. 2007), and as much as 30\% during a 2-min maximal contraction (Taylor et al. 2006). Overall, fatigue has clear implications to physical performance, and is experienced as a chronic activity-limiting symptom that adversely affects the quality of life in many physical, neurological and psychiatric disorders. However, exactly how brain activity is modulated during physical fatigue is not well understood.

Previous attempts at neuroimaging during physical fatigue have largely relied upon functional magnetic resonance imaging (fMRI). Here, an increase in sensorimotor neural activity is inferred from increases in the blood-oxygen-level dependent (BOLD) fMRI signal, which have been found to accompany fatigue onset during the performance of both maximal (Liu et al. 2002; Steens et al. 2012) and submaximal isometric contractions (Liu et al. 2003; van Duinen et al. 2007; Benwell et al. 2007). However, the haemodynamically derived fMRI signal is both physiologically and temporally remote from the electrical activity that is of primary interest as the method of communication within the brain. Dendritic currents, synchronised across neuronal assemblies, manifests as local oscillations, which have been shown to play an important 
role in co-ordinating brain function (Donner \& Siegel 2011). This rhythmic oscillatory activity can be non-invasively assessed using electroencephalography (EEG) and magnetoencephalography (MEG), with the latter offering greater spatial resolution and sensitivity due, in part, to the fact that magnetic fields are not distorted by the biological tissues between the cortex and sensors (Cheyne 2013).

In the sensorimotor system, motor action has been linked with robust changes in neural oscillations in the mu $(\sim 8-15 \mathrm{~Hz})$ and beta $(\sim 15-30 \mathrm{~Hz})$ bands. During the preparation and performance of unilateral movements, decreases in both mu and beta amplitude are observed, with the largest effect occurring local to the contralateral primary sensorimotor cortex (Jasper \& Penfield 1949; Salmelin \& Hari 1994; Pfurtscheller et al. 2003). These responses are known as the movement-related mu/beta decrease (MRMD/MRBD), or event-related desynchronisation. Following movement cessation, beta oscillations exhibit a period of elevated amplitude, known as the post-movement beta rebound (PMBR) (Pfurtscheller et al. 1996; Jurkiewicz et al. 2006). These responses have been measured widely, and recent work suggests that they may have great potential to inform important biomarkers for disease. For example, PMBR is greater in healthy controls than patients with schizophrenia, and the magnitude of PMBR in patients correlates with persistent symptoms of disease (Robson et al. 2016). In individuals with Parkinson's disease, where movements are limited and poorly controlled, resting beta oscillations are increased and the relative magnitude of MRBD and PMBR are reduced in amplitude compared with healthy controls (HeinrichsGraham et al. 2014; Pollok et al. 2012). Additionally, these responses may have further clinical utility by informing brain computer interfaces (Pfurtscheller \& Solis-Escalante 2009). 
Despite their robust nature and high potential value these movement-related oscillatory responses remain poorly understood, and whether they are modulated by physical fatigue is largely unknown. Knowledge of the interactions between sensorimotor oscillatory activity and physical fatigue may help to inform our understanding of the nature of fatigue. Moreover, these interactions may have important implications for researchers who measure and interpret these oscillatory responses in a wider context.

One preliminary study measured sensorimotor oscillations during submaximal contractions performed in a state of physical fatigue and reported an elimination of MRBD (Tecchio et al. 2006). This might indicate a strong influence of fatigue on the typically robust movement-related oscillatory dynamics described above, importantly however, no control session was conducted making it impossible to isolate fatigue from time/habituation effects. Therefore, the purpose of the current study was to determine the influence of physical fatigue on movement-related oscillatory dynamics within the sensorimotor cortex, using a crossover (fatigue and control) design to isolate the effects of fatigue. Our previous work (Fry et al. 2016) has shown MRBD magnitude to be independent of changes in contraction kinetics, while PMBR was augmented following greater sensorimotor activity. By extension, we hypothesise that MRMD and MRBD will be unaffected by the development of physical fatigue, whereas the PMBR following submaximal contractions may be increased.

\section{MATERIALS AND METHODS}

\section{Participants}


Fourteen healthy adults with no known history of neurological or musculoskeletal disorders volunteered their participation. Three participants found the fatigue protocol (described below) particularly challenging, such that they were unable to maintain the post-intervention test contractions for the prescribed time, and were omitted from subsequent analyses. This left a total of eleven participants ( 7 males, 2 left handed, age $27 \pm 4$ years [mean \pm standard deviation]). The experimental procedures were approved by the Loughborough University Ethical Advisory Committee, and each participant provided written informed consent prior to their involvement. All experimental measurements were carried out in the MEG facility at the Sir Peter Mansfield Imaging Centre, University of Nottingham, UK.

\section{Experimental protocol}

An overview of the experimental procedure is given in Fig. 1. Participants were seated upright in the MEG system with their right forearm and hand positioned in a custom built isometric wrist-flexion dynamometer that was secured to the armrest of the MEG system (Fig. 1a,b). The dynamometer held the participant's arm in a neutral position of pronation/supination, radial/ulnar deviation and wrist flexion/extension. Waist and right forearm straps were lightly applied to maintain a consistent posture, but without risk of restricting blood flow. Participants were instructed to exert wrist-flexion force against a cylindrical handle that was attached in series to a strain gauge. The handle was sewn onto a mitt that participants wore throughout the experimental sessions to ensure a consistent position of the handle relative to the hand (and therefore a constant lever 
length). Participants were also instructed to refrain from any movements other than the prescribed wrist-flexion (e.g. gripping).

Participants were well familiarised with the motor tasks and MEG environment prior to their participation in two experimental sessions. A randomised crossover design was adopted with sessions completed approximately 7 days apart and at a consistent time of day. Participants were instructed to abstain from strenuous or atypical exercise for 36 hours prior to each experimental session, and to avoid the intake of nutritional stimulants (e.g. caffeine) within two hours of the session.

Each experimental session started with a short warm up of submaximal isometric wristflexions. Participants then completed four maximal voluntary contractions, with $30 \mathrm{~s}$ rest between contractions, from which a baseline value of maximal voluntary force $\left(\mathrm{MVF}_{\mathrm{b}}\right)$ was established. Participants were instructed to exert a maximum effort of wrist-flexion force continuously for $3 \mathrm{~s}$, with visual biofeedback and verbal encouragement provided. The peak force (200 ms epoch) during these contractions was used as the measure of $\mathrm{MVF}_{\mathrm{b}}$ from which all subsequent force outputs were prescribed. A 5-min rest was then provided before commencing the pre-intervention test contractions.

Pre- and post-intervention test contractions both involved an identical series of fourteen prescribed wrist-flexion contractions: two maximal voluntary contractions $\left(\mathrm{MVF}_{\text {start }}\right)$, ten contractions at $40 \% \mathrm{MVF}_{\mathrm{b}}$, and a further two maximal contractions ( $\mathrm{MVF}_{\text {end }}$ ) (Fig. 1d). The $40 \% \mathrm{MVF}_{\mathrm{b}}$ contractions were $6 \mathrm{~s}$ duration, and performed every $30 \mathrm{~s}$, in order to measure oscillatory activity with MEG both during (MRMD \& MRBD) and post contraction (PMBR). These MEG recordings provided the primary outcome measures to 
test the neuronal responses before and after the fatigue/control interventions. Submaximal contractions were used so that participants were able to repeat the contractions enough times to collect a sufficient quantity of MEG data, and also to ensure that the same motor task (with respect to force output) could be replicated even during a state of physical fatigue. The maximal voluntary contractions were used to determine MVF at two time-points pre- and post-intervention, allowing monitoring of each participant's fatigue throughout each experimental session. These maximal contractions were 3 s duration, and performed every 30 s. Participants had received prior instructions to perform a maximal effort of wrist-flexion for this entire duration.

Both interventions comprised a series of 40 contractions; each of $12 \mathrm{~s}$ duration, performed every $30 \mathrm{~s}$, and at a constant-force of either $5 \% \mathrm{MVF}_{\mathrm{b}}$ (control intervention) or $55 \% \mathrm{MVF}_{\mathrm{b}}$ (fatigue intervention). An additional $90 \mathrm{~s}$ of rest was provided both before and after the intervention. The fatigue intervention was designed to induce physical fatigue, whereas the control intervention was designed to involve the same number and duration of contractions as the fatigue intervention, but without posing a physical challenge. As fatigue developed during the fatigue intervention, participants attempted to maintain the $55 \% \mathrm{MVF}_{\mathrm{b}}$ force output for as much of the $12 \mathrm{~s}$ as they could, and maintained a maximal effort of force output thereafter. The specific force level for the fatigue intervention $\left(55 \% \mathrm{MVF}_{\mathrm{b}}\right)$ was selected following comprehensive pilot testing, which found this protocol to be practicable for most participants, while also being fatiguing to all those who attempted it.

Force prescription and feedback was facilitated by participants viewing a visual display throughout the experimental procedures. They were presented with a temporal profile of target force output prior to each of the prescribed contractions and attempted to match 
their contraction force to the target profiles as closely as possible. Real-time (measured) contraction force was overlaid on the target profile and this provided feedback (see Fig. 1c). The target force profile for each contraction included a preparatory period of 2-8 s at $0 \% \mathrm{MVF}_{\mathrm{b}}$ (time dependent on forthcoming contraction duration), a linear ramp of $1 \mathrm{~s}$ duration, and a constant-force (plateau) phase at the prescribed force output (times detailed above). Each profile disappeared to leave a blank screen immediately following each contraction (constant-force phase). Participants received prior instructions to cease the contraction as soon as the target force profile had disappeared from the screen.

\section{Data Collection}

MEG data were collected during the $40 \% \mathrm{MVF}_{\mathrm{b}}$ test contractions and intervening rest periods. MEG data were sampled at $600 \mathrm{~Hz}$ using a 275 channel CTF MEG system (MISL, Coquitlam, Canada) operating in third order synthetic gradiometer configuration. Three localisation coils were attached to the head as fiducial markers (nasion, left preauricular and right preauricular) prior to the recording. Energising these coils throughout data acquisition enabled continuous localisation of the fiducial markers relative to the MEG sensor geometry. This also allowed us to monitor if the participants' head position had shifted during the intervention, and to make readjustments prior to the post-intervention test contractions. In order to coregister individual brain anatomy to the MEG sensor array, each participant's head shape was digitised (Polhemus IsoTrack, Colchester, VT, USA) relative to the fiducial markers prior to the MEG recording. Separately, volumetric anatomical MR images were acquired for each participant using a 3 T MR system (Phillips Achieva, Best, Netherlands) running an MPRAGE sequence 
$\left(1-\mathrm{mm}^{3}\right.$ resolution). Each participant's head surface was extracted from the MR image and coregistered (via surface matching) to their digitised head shape. This allowed complete coregistration of the MEG sensor array to the brain anatomy, facilitating subsequent forward and inverse calculations.

Force data were measured using a calibrated S-beam strain gauge (0-500 N linear range; Force Logic, Swallowfield, UK) housed in the isometric wrist-flexion dynamometer. Force data were sampled at $2000 \mathrm{~Hz}$ by a PC running Spike 2 software (CED, Cambridge, UK), via an external A/D converter (Micro 1401, CED, Cambridge, UK). For each individual contraction, markers were inserted within the MEG and force recordings to time-synchronise the two data sources.

\section{Data Analyses}

Mean force output and steadiness (standard deviation of the contraction force within each $40 \% \mathrm{MVF}_{\mathrm{b}}$ contraction) were determined for each individual contraction. Averages of these mean and steadiness values were calculated first within each individual, and subsequently averaged across participants. The first and last $0.5 \mathrm{~s}$ of each contraction (constant-force phase) were excluded from all analyses to ensure force output was at the prescribed level throughout the analysed time-window (see Fig. 1e).

Initially, MEG data were visually inspected in order to exclude common sources of interference, including the magnetomyogram, magnetooculogram and magnetocardiogram, which are easily identified from their well characterised MEG signatures. Following this pre-processing, MEG data were analysed using synthetic aperture magnetometry (SAM) (Vrba \& Robinson 2001), a beamforming variant used to 
localise neural oscillatory amplitude changes. Firstly, pre- and post-intervention measurements were concatenated so that source localisation produced a single location of interest (LOI) for each experimental session. This approach ensured the region of the cortex analysed was consistent pre- and post-intervention, and any observed changes in oscillatory amplitudes were due to the intervention and not an inconsistency in LOI. Subsequently, data were filtered to the mu $(8-15 \mathrm{~Hz})$ and beta $(15-30 \mathrm{~Hz})$ bands. These exact frequency bands were iteratively determined by generating time-frequency spectra and evaluating the banded oscillatory responses. Importantly, the $15 \mathrm{~Hz}$ boundary was chosen to optimally dissociate the mu and beta responses. Oscillatory amplitude was then contrasted between active and control time windows in order to delineate the spatial signatures of mu and beta amplitude changes. Individual LOIs were sought for the MRMD, MRBD and PMBR in order to construct beamformers that were optimal for quantifying each response, and because the peak location of these phenomena may differ significantly (Fry et al. 2016). To localise MRMD and MRBD an active window between 0.5 and $5.5 \mathrm{~s}$ of the $40 \% \mathrm{MVF}_{\mathrm{b}}$ test contractions was used. To localise PMBR an active window commencing $0.25 \mathrm{~s}$ after contraction offset and lasting $5 \mathrm{~s}$ was used; where contraction offset was defined as the time at which contraction force fell below $2 \% \mathrm{MVF}_{\mathrm{b}}$ when returning to rest (Fig. 1e). In all cases, the control window commenced $20 \mathrm{~s}$ after the prescribed contraction onset and lasted for $5 \mathrm{~s}$. The forward model was based upon a multiple local sphere head model and the forward calculation by Sarvas (Sarvas 1987; Huang et al. 1999). Pseudo-t-statistical images (5-mm ${ }^{3}$ isotropic resolution) were generated showing regions of oscillatory amplitude exhibiting significant $(\mathrm{P} \leq 0.05)$ change in the mu and beta bands. Spatial peaks occurring within 
sensorimotor regions were identified (where present) and used as LOIs for subsequent analysis.

Following identification of LOIs using SAM, time frequency spectrograms were generated for each individual participant in order to measure oscillatory dynamics both pre- and post-intervention, in both sessions. Here, another SAM beamformer was applied, this time with weighting parameters determined for each LOI using a covariance window spanning the $1-150 \mathrm{~Hz}$ frequency range, and a time window encompassing the ten $40 \% \mathrm{MVF}_{\mathrm{b}}$ test contractions and their inter-contraction rest periods. The derived beamformer weights for each location were multiplied by the MEG data (filtered 1-150 Hz) to create a 'virtual sensor' time-series of the electrical activity at that LOI. Virtual sensor time-series were filtered into 31 overlapping frequency bands, and a Hilbert transform was used to generate the amplitude envelope of oscillations within each band. For each intervention (fatigue and control), these envelope time-courses were then averaged across the ten $40 \% \mathrm{MVF}_{\mathrm{b}}$ test contractions measured pre- and post-intervention, separately. Averaged envelopes were baseline corrected by subtracting the baseline activity (average signal from 20-25 s window relative to contraction onset) from the whole 25-s trial, for each envelope separately. Baseline corrected envelopes in the overlapping frequency bands were then concatenated in the frequency dimension to generate a single time frequency spectrum (TFS) per participant, for each LOI identified. TFSs were subsequently averaged over all participants leaving a single TFS from the spatial maxima of the MRMD, MRBD and PMBR.

To assess the effect of fatigue on oscillatory activity, mean values of MRMD, MRBD and PMBR were extracted from the TFS data for each participant. MRMD and MRBD 
were calculated as the integral of the amplitude of the signal in the $8-15 / 15-30 \mathrm{~Hz}$ frequency bands across the same active time windows as those used to identify LOIs (0.5-5.5 s relative to contraction onset), and were divided by the duration ( $5 \mathrm{~s})$ of these windows. Thus, MRMD and MRBD represent the mean mu/beta amplitude decrease from baseline during the $40 \% \mathrm{MVF}_{\mathrm{b}}$ test contractions. For PMBR, the total integral of the beta amplitude was calculated between 0-10 s following contraction offset, which allowed for this protracted response to reach baseline. These results were separately generated from the individual LOIs of each response in each participant. This analysis yielded a single value (absolute difference from baseline) of MRMD, MRBD and PMBR for each participant, both pre- and post-intervention in both the fatigue and control sessions (i.e. four values per participant). These values were then averaged across participants to determine group means and standard deviations. In addition, MRMD, MRBD and PMBR were also calculated as percentage changes from baseline amplitude to investigate whether any changes in these oscillatory responses can be explained purely by changes in baseline activity.

Individual responses were confirmed as local to the contralateral sensorimotor cortex in individual brain space following SAM beamforming, however, to characterise the group mean spatial location of MRMD, MRBD and PMBR, individual brain images were normalised to an anatomical standard (Montreal Neurological Institute (MNI) brain) using FLIRT in FSL. The MNI coordinates for each peak (MRMD, MRBD and PMBR) in each participant were then measured, before averaging across participants to create MNI co-ordinates of the group means. The most likely cortical locations of the mean coordinates were then determined using the Oxford-Harvard brain atlas. 
Two-way repeated measures ANOVAs were used to compare both force output and neural oscillations between experimental sessions (control vs. fatigue session) and across measurement time-points (e.g. pre- vs. post-intervention for neural oscillations, or 4 time-points [start and end of test contractions both pre- and post-intervention] for MVF). In the event of a significant session $\times$ time-point interaction effect, post-hoc paired t-tests/ one-way repeated measures ANOVAs (with Bonferroni corrections) were performed as appropriate to elucidate the cause of these effects. Effect sizes (Cohen's $d_{z}$ [as per Lakens (2013)]) were also calculated using the delta scores (post - pre) for the control and fatigue sessions where a significant interaction effect was identified in the analyses of MRMD, MRBD and PMBR. Finally, a paired t-test was also used to compare the average contraction force between the first and last five repetitions of the fatigue intervention. A P-value below 0.05 was considered statistically significant. Data are expressed as group means \pm standard error of the mean (SEM) unless otherwise stated.

\section{RESULTS}

\section{Force measurements}

Eleven participants were able to accurately perform the prescribed $40 \% \mathrm{MVF}_{\mathrm{b}}$ test contractions throughout both experimental sessions (Table 1). Neither intervention had any influence on either the mean force output (ANOVA; session, time-point and session $\times$ time-point interaction effects: all $\mathrm{P} \geq 0.269$ ) or the steadiness of force output (all $\mathrm{P} \geq 0.096$ ) during the $40 \% \mathrm{MVF}_{\mathrm{b}}$ contractions. Thus, the motor task during which 
oscillatory neuronal activity was assessed was kinetically equivalent before and after both interventions.

Wrist-flexion $\mathrm{MVF}_{\mathrm{b}}$ was similar for the fatigue and control sessions (mean \pm standard deviation: $282.8 \pm 82.6 \mathrm{~N}$ and $278.1 \pm 74.4 \mathrm{~N}$, respectively). During the fatigue intervention, maintaining $55 \% \mathrm{MVF}_{\mathrm{b}}$ for $12 \mathrm{~s}$ became supra-maximal for all participants. Overall, the mean force was $23.0 \%$ lower in the last five contractions compared to the first five (of forty) $\left(53.7 \pm 0.5\right.$ vs. $41.3 \pm 2.2 \% \mathrm{MVF}_{\mathrm{b}}$; t-test, $\left.\mathrm{P}<0.001\right)$, demonstrating the occurrence of fatigue during this intervention. Conversely, the $5 \% \mathrm{MVF}_{\mathrm{b}}$ contractions in the control intervention were performed without any difficulty or sensations of fatigue.

The efficacy of the fatigue intervention was clearly demonstrated by the MVF measurements (ANOVA; session $\times$ time-point interaction effect: $\mathrm{P}<0.001$; Fig. 2). Both $\mathrm{MVF}_{\text {post-start }}$ and $\mathrm{MVF}_{\text {post-end }}$ (see Fig. 1d) were markedly attenuated from their respective pre-intervention values $\left(\mathrm{MVF}_{\text {pre-start }}\right.$ and $\left.\mathrm{MVF}_{\text {pre-end }}\right)$ in the fatigue session ($29.5 \pm 3.0 \%$ and $-28.9 \pm 2.4 \%$, ANOVA: $\mathrm{P}<0.001$ following Bonferroni correction) but not the control session $(\mathrm{P} \geq 0.511)$. Additionally, paired t-tests confirmed that MVF differed between the two experimental sessions in both post-intervention measurements $(\mathrm{P}<0.001)$, but not pre-intervention $(\mathrm{P} \geq 0.531)$. No significant differences were observed between $\mathrm{MVF}_{\text {start }}$ and $\mathrm{MVF}_{\text {end }}$ either pre- or post-intervention in either session ( $\mathrm{P} \geq 0.063$ ), indicating that the $40 \% \mathrm{MVF}_{\mathrm{b}}$ test contractions did not have a significant fatiguing effect in themselves.

\section{MEG measurements}


The primary results for the mu band analyses are shown in Fig. 3 A clear MRMD, local to the contralateral sensorimotor cortex, was observed in 9 of the 11 participants. Fig. 3a illustrates the locations of peak MRMD for these 9 participants, plotted in MNI space. Fig. $3 \mathrm{~b}$ shows the magnitude of the baseline (resting) mu amplitude at the location of MRMD, averaged across participants, both pre- and post-intervention in both sessions. Statistical analysis revealed that resting mu amplitude was similar both pre- and postintervention in both experimental sessions (ANOVA; session, time-point and session $\times$ time-point interaction effects: all $\mathrm{P} \geq 0.092$; Fig. 3b). Fig. $3 \mathrm{c}$ shows the time-frequency spectrograms extracted from the locations of MRMD, and averaged across participants. In each TFS, blue represents a decrease in oscillatory amplitude with respect to baseline whereas yellow reflects an increase. A clear decrease in mu oscillations (the MRMD) occurring prior to and throughout the $40 \% \mathrm{MVF}_{\mathrm{b}}$ contractions is seen in each TFS. Fig. $3 d$ shows the average magnitude of MRMD. MRMD was similar throughout both experimental sessions (ANOVA; session, time-point and session $\times$ time-point interaction effects: all $\mathrm{P} \geq 0.325$; Fig. $3 \mathrm{~d}$ ), suggesting that there was no consistent effect of physical fatigue on mu band oscillatory dynamics.

The primary results for the beta band analyses are shown in Fig. 4 and 5. The layouts are equivalent to that of Fig. 3 (described above). A clear MRBD and PMBR, local to the contralateral sensorimotor cortex, was observed in 10 and 9 of the 11 participants, respectively. The locations of peak MRBD and PMBR for all participants are shown in Figs. 4a and 5a. Resting beta activity at the location of MRBD decreased between preand post-intervention (ANOVA, time-point effect: $\mathrm{P}=0.014$; Fig. 4b), however these changes were similar in both sessions (session and session $\times$ time-point interaction effects: both $\mathrm{P} \geq 0.278$ ). Resting beta amplitude at the location of PMBR also decreased 
following both interventions, and with no difference between experimental sessions (ANOVA, time-point effect: $\mathrm{P}=0.031$; session and session $\times$ time-point interaction effects: both $\mathrm{P} \geq 0.138$; Fig. $5 b$ ). As expected there was a decrease in beta amplitude immediately prior to and throughout the $40 \% \mathrm{MVF}_{\mathrm{b}}$ contractions, and a beta rebound following contraction offset was evident during both experimental sessions (Fig. 4c and 5c). MRBD demonstrated a different response to the two interventions (ANOVA, session $\times$ time-point interaction: $\mathrm{P}=0.021$; effect size: $d_{\mathrm{z}}=0.89$; Fig. $4 \mathrm{~d}$ ); decreasing in magnitude from pre- to post-intervention in the control session (i.e. a smaller decrease in beta amplitude is seen post-control intervention; $t$-test: $\mathrm{P}=0.006$ ), but not the fatigue session $(\mathrm{P}=0.470)$. Conversely, the magnitude of PMBR was affected by physical fatigue (ANOVA; session $\times$ time-point interaction: $\mathrm{P}=0.018$; effect size: $d_{\mathrm{z}}=0.99$; Fig. 5d); demonstrating an increase following the fatigue intervention (i.e. a larger PMBR post-fatigue intervention; $\mathrm{t}$-test: $\mathrm{P}<0.001)$, but not the control intervention $(\mathrm{P}=0.623)$.

The mean MNI coordinates of MRMD, MRBD and PMBR are displayed in Table 2, alongside the most likely cortical locations of these coordinates according to the Oxford-Harvard brain atlas. These results show the spatial peaks of MRMD and MRBD were located postcentrally, whereas PMBR arose precentrally.

Overall, whether MRMD, MRBD and PMBR were expressed in absolute terms (presented above), or as a percentage change relative to resting (baseline) oscillatory amplitude, the effects of physical fatigue on these oscillatory responses were extremely similar. Relative MRBD showed a session $\times$ time-point interaction, with a substantial decrease after the control intervention and no change following the fatigue intervention (Table 3). This was despite the decrease in resting beta amplitude observed in both sessions. In addition, relative PMBR also demonstrated a tendency for a session $\times$ time- 
point interaction for relative PMBR, with a greater rebound observed post-fatigue but not post-control.

\section{DISCUSSION}

Physical fatigue arises not only from peripheral processes within the muscles but also from supraspinal mechanisms within the cortex. However, exactly how electrophysiological brain activity is modulated during fatigue is not well understood. This study used MEG to provide a direct measure of neural activity during voluntary muscle contractions performed in a state of physical fatigue. The primary findings were that MRBD was maintained following the fatigue inducing intervention, in contrast a reduction in MRBD was found after the control intervention, and PMBR magnitude was found to increase following the fatigue but not the control intervention. MRMD was unchanged following either intervention.

From the force recordings it was clear that the fatigue intervention induced substantial physical fatigue $(\sim 30 \%$ reduction in $\mathrm{MVF})$ that endured throughout the postintervention measurements; and was in clear contrast to the control session. Moreover, despite the exhibited fatigue, eleven of the fourteen initial participants were able to accurately perform all of the prescribed $40 \% \mathrm{MVF}_{\mathrm{b}}$ contractions, which were used to assess movement-related oscillatory dynamics. These contractions were kinetically equivalent (force and steadiness) before and after both interventions, demonstrating performance of a consistent task despite fatigue. 
The MRBD was consistent throughout the fatigue session, whereas an attenuation was observed following the control intervention. Our observation that MRBD was maintained following the fatigue intervention is in contrast to one previous study that reported an elimination of MRBD during submaximal contractions of the extensor communis digitorum performed after a prolonged maximal voluntary contraction of the same muscle (Tecchio et al. 2006). However, this study lacked both a control session and an objective measure of physical fatigue. In the current investigation, the presence of fatigue throughout the post-intervention measurements was clearly demonstrated by the $\sim 30 \%$ decrease in MVF, which was not the case for the control session. Furthermore, these authors (Tecchio et al. 2006) found agonist electromyography amplitude during the submaximal contractions to be unaffected by the intervention, in contrast to the widely documented increase in electromyography amplitude that occurs with fatigue (Bigland-Ritchie et al. 1986; Dorfman et al. 1990).

In general, high amplitude beta oscillations are widely believed to reflect cortical inhibition (Cassim et al. 2001; Gaetz et al. 2011). For example, blocking reuptake of the inhibitory neurotransmitter gamma aminobutyric acid (GABA) via administration of Tiagabine increases both baseline beta amplitude and MRBD (Muthukumaraswamy et al. 2013). Administration of diazepam (a GABA-A receptor modulator) has also indicated MRBD to be a GABA-A mediated process (Hall et al. 2011). Thus, the MRBD likely reflects a removal of inhibition during movement planning and execution, enabling a greater flexibility for local encoding processes within cellular assemblies (Donner \& Siegel 2011; Brookes et al. 2015). Furthermore, a task habituation effect of attenuated MRBD has been found to occur during the early phases of task repetition (Kranczioch et al. 2008; Studer et al. 2010; Pollok et al. 2014) and may be a 
neurophysiological marker of early cortical reorganisation with task habituation (Pollok et al. 2014). Therefore, the decrease in MRBD between pre- and post-intervention in the control session of the current study adds to the weight of evidence that task habituation attenuates MRBD and may reflect a reduction in the number of sensorimotor neural populations recruited to perform a given task following habituation (Mancini et al. 2009). The current study also extends previous work by showing that this habituation effect of attenuated MRBD was counteracted by the induction of physical fatigue. Fatigue is known to induce an extensive range of neurophysiological adaptations (see Gandevia 2001) including an increased corticospinal output to maintain a consistent force output, and also induces extensive group III and IV afferent feedback in response to metabolite accumulation (Taylor \& Gandevia 2008). These adaptations may have meant that, from a cortical perspective at least, the task of performing the prescribed $40 \% \mathrm{MVF}_{\mathrm{b}}$ contractions remained unfamiliarised (i.e. participants effectively remained non-habituated).

To the authors' knowledge, this was the first study to investigate the effect of physical fatigue on PMBR. The results demonstrated that PMBR was augmented following the fatigue intervention but not the control intervention. The increase in beta amplitude following movement cessation is believed to reflect an active inhibition of the sensorimotor networks recruited during the preparation and execution phases of motor actions (Alegre et al. 2008; Solis-Escalante et al. 2012). Moreover, there is growing evidence to suggest that beta modulations, and PMBR in particular, are related to GABAergic inhibition. Specifically, the magnitude of PMBR has been shown to correlate with individuals' GABA levels, measured using magnetic resonance spectroscopy (Gaetz et al. 2011). Although administration of a $\mathrm{GABA}_{\mathrm{A}}$ receptor 
modulator indicated PMBR was a non-GABA $\mathrm{A}_{\mathrm{A}}$ mediated process (Hall et al. 2011). Therefore, a greater PMBR during fatigue might reflect a stronger $\mathrm{GABA}_{\mathrm{B}}$-driven inhibition of sensorimotor networks that were more highly activated/excited during contraction; potentially due to increases in corticospinal output or somatosensory processing (either directly or indirectly). For example, the magnitude of the PMBR may be positively related to the number of motor units recruited during voluntary contractions (Pfurtscheller et al. 1998; Fry et al. 2016), which is known to increase with the development of fatigue (Bigland-Ritchie et al. 1986). Alternatively, Cassim et al. (2001) proposed that movement-related somatosensory processing may be fundamental to the engenderment of the PMBR, having observed a PMBR to be present following passive finger movements but not active movements performed under ischaemic deafferentation. Therefore, increased activity of fatigue sensitive afferents may also have contributed to the larger PMBR observed following the development of physical fatigue.

That PMBR may be modulated by fatigue has wide implications for the measurement and interpretation of this response. Recent work has demonstrated that PMBR has the potential to be used as a biomarker for pathology, with examples including Parkinson's disease (Pollok et al. 2012; Heinrichs-Graham et al. 2014; Hall et al. 2014) and schizophrenia (Robson et al. 2016). Judicious design of study protocols is therefore essential to ensure the PMBR is not affected by the experimental procedures, particularly when investigating disorders in which susceptibility to fatigue represents a common symptom.

Resting beta amplitude at the locations of both MRBD and PMBR demonstrated similar decreases following both interventions. De Pauw et al. (2013) noted a decreased resting 
beta activity across the whole brain after a prolonged intensive cycling performance in the heat; designed to induce supraspinal fatigue. Additionally, Jagannath \& Balasubramanian (2014) found widespread decreases in beta amplitude following a monotonous 60-min simulated driving task, which was designed to induce mental fatigue. In the present study, the decrease in resting beta amplitude following the interventions was not specific to the fatigue session; however, other fatigue modalities, such as mental fatigue arising from the prolonged periods of concentration and task repetition, might have contributed to this beta decrease. While this reduction in baseline beta activity may contributed to the observed changes in MRBD and PMBR, these changes cannot completely explain the attenuated MRBD following the control intervention and increased PMBR following the fatigue intervention (see Table 3).

Fatigue had no effect on either resting mu amplitude or MRMD, which were both unchanged following both interventions. This indicated that mu activity within the sensorimotor cortex was not affected by either the induction of physical fatigue, or the prolonged period of task adherence involved in both experimental sessions.

In conclusion, this study revealed two novel findings regarding the effects of physical fatigue on movement-related sensorimotor oscillatory dynamics. Firstly, physical fatigue offsets the attenuation in MRBD observed with repetition of a motor task in a non-fatiguing control session. Secondly, PMBR increased when submaximal contractions were performed in a state of physical fatigue; which supports an emerging theory that PMBR is sensitive to increases in corticospinal output and changes in sensory input, both of which would be expected to occur with physical fatigue. 


\section{ACKNOWLEDGEMENTS}

This work was funded by a Medical Research Council New Investigator Research Grant (MR/M006301/1) awarded to MJB. AF was funded by a Loughborough University Graduate School studentship. GCO is funded by an MRC studentship. KJM is funded by an Anne McLaren Fellowship. We also acknowledge Medical Research Council Partnership Grant (MR/K005464/1).

\section{CONFLICTS OF INTEREST}

There are no competing interests to declare.

\section{REFERENCES}

Alegre, M., Alvarez-Gerriko, I., Valencia, M., Iriarte, J. \& Artieda, J. 2008. Oscillatory changes related to the forced termination of a movement. Clin Neurophysiol 119, $290-300$

Benwell, N., Mastaglia, F. \& Thickbroom, G. 2007. Changes in the functional MR signal in motor and non-motor areas during intermittent fatiguing hand exercise. Exp Brain Res 182, 93-97.

Bigland-Ritchie, B., Cafarelli, E. \& Vøllestad, N. 1986. Fatigue of submaximal static 
contractions. Acta Physiol Scand Suppl 556, 137-148.

Brookes, M., Hall, E., Robson, S., Price, D., Palaniyappan, L., Liddle, E., Liddle, P., Robinson, S. \& Morris, P. 2015. Complexity measures in magnetoencephalography: Measuring “disorder” in schizophrenia. PLoS One 10, $\mathrm{e} 0120991$.

Cassim, F., Monaca, C., Szurhaj, W., Bourriez, J.-L., Defebvre, L., Derambure, P. \& Guieu, J-D. 2001. Does post-movement beta synchronization reflect an idling motor cortex? Neuroreport 12, 3859-3863.

Cheyne, D. 2013. MEG studies of sensorimotor rhythms: a review. Exp Neurol 245, 2739.

De Pauw, K., Roelands, B., Marusic, U., Tellez, H.F., Knaepen, K. \& Meeusen, R. 2013. Brain mapping after prolonged cycling and during recovery in the heat. $J$ Appl Physiol 115, 1324-1331.

Donner, T. \& Siegel, M. 2011. A framework for local cortical oscillation patterns. Trends Cogn Sci 15, 191-199.

Dorfman, L., Howard, J. \& McGill, K. 1990. Triphasic behavioral response of motor units to submaximal fatiguing exercise. Muscle and Nerve 13, 621-628.

Fry, A., Mullinger, K., O’Neill, G., Barratt, E., Morris, P., Bauer, M., Folland, J. \& Brookes, M. 2016. Modulation of post-movement beta rebound by contraction force and rate of force development. Hum Brain Mapp doi:10.1002/hbm.23189

Gaetz, W., Edgar, J., Wang, D. \& Roberts, T. 2011. Relating MEG measured motor 
cortical oscillations to resting $\gamma$-aminobutyric acid (GABA) concentration. Neuroimage 55, 616-621.

Gandevia, S. 2001. Spinal and supraspinal factors in human muscle fatigue. Physiol Rev $81,1725-1789$.

Hall, S., Prokic, E., McAllister, C., Ronnqvist, K., Williams, A., Yamawaki, N., Witton, C., Woodhall, G. \& Stanford, I. 2014. GABA-mediated changes in interhemispheric beta frequency activity in early-stage Parkinson's disease. Neuroscience 281, 68-76.

Hall, S., Stanford, I., Yamawaki, N., Mcallister, C., Rönnqvist, K., Woodhall, G. \& Furlong, P. 2011. The role of GABAergic modulation in motor function related neuronal network activity. Neuroimage 56, 1506-1510.

Heinrichs-Graham, E., Wilson, T., Santamaria, P., Heithoff, S., Torres-Russotto, D., Hutter-Saunders, J., Estes, K., Meza, J., Mosley, R. \& Gendelman, H. 2014. Neuromagnetic evidence of abnormal movement-related beta desynchronization in Parkinson's disease. Cereb Cortex 24, 1-10.

Huang, M., Mosher, J. \& Leahy, R. 1999. A sensor-weighted overlapping-sphere head model and exhaustive head model comparison for MEG. Phys Med Biol 44, 423440.

Jagannath, M. \& Balasubramanian, V. 2014. Assessment of early onset of driver fatigue using multimodal fatigue measures in a static simulator. Appl Ergon 45, 11401147.

Jasper, H. \& Penfield, W. 1949. Electrocorticograms in man: Effect of voluntary 
movement upon the electrical activity of the precentral gyrus. Arch für Psychiatr und Zeitschrift Neurol 183, 163-174.

Jurkiewicz, M., Gaetz, W., Bostan, A. \& Cheyne, D. 2006. Post-movement beta rebound is generated in motor cortex: Evidence from neuromagnetic recordings. Neuroimage 32, 1281-1289.

Kranczioch, C., Athanassiou, S., Shen, S., Gao, G. \& Sterr, A. 2008. Short-term learning of a visually guided power-grip task is associated with dynamic changes in EEG oscillatory activity. Clin Neurophysiol 119, 1419-1430.

Lakens, D. 2013. Calculating and reporting effect sizes to facilitate cumulative science: A practical primer for t-tests and ANOVAs. Frontiers in Psychology 4, 863.

Liu, J., Dai, T., Sahgal, V., Brown, R. \& Yue, G. 2002. Nonlinear cortical modulation of muscle fatigue: a functional MRI study. Brain Res 957, 320-329.

Liu, J.Z., Shan, Z.Y., Zhang, L.D., Sahgal, V., Brown, R.W. \& Yue, G.H. 2003. Human brain activation during sustained and intermittent submaximal fatigue muscle contractions: an FMRI study. J Neurophysiol 90, 300-312.

Mancini, L., Ciccarelli, O., Manfredonia, F., Thornton, J., Agosta, F., Barkhof, F., Beckmann, C., De Stefano, N., Enzinger, C., Fazekas, F., Filippi, M., Gass, A., Hirsch, J., Johansen-Berg, H., Kappos, L., Korteweg, T. et al. 2009. Short-term adaptation to a simple motor task: A physiological process preserved in multiple sclerosis. Neuroimage 45, 500-511.

Muthukumaraswamy, S., Myers, J., Wilson, S., Nutt, D., Lingford-Hughes, A., Singh, K. \& Hamandi, K. 2013. The effects of elevated endogenous GABA levels on 
movement-related network oscillations. Neuroimage 66, 36-41.

Pfurtscheller, G., Graimann, B., Huggins, J., Levine, S. \& Schuh, L. 2003. Spatiotemporal patterns of beta desynchronization and gamma synchronization in corticographic data during self-paced movement. Clin Neurophysiol 114, 12261236.

Pfurtscheller, G. \& Solis-Escalante, T. 2009. Could the beta rebound in the EEG be suitable to realize a "brain switch"? Clin Neurophysiol 120, 24-29.

Pfurtscheller, G., Stancák, A. \& Neuper, C. 1996. Post-movement beta synchronization. A correlate of an idling motor area? Electroencephalogr Clin Neurophysiol 98, 281-293.

Pfurtscheller, G., Zalaudek, K. \& Neuper, C. 1998. Event-related beta synchronization after wrist, finger and thumb movement. Electroencephalogr Clin Neurophysiol $109,154-160$.

Pollok, B., Krause, V., Martsch, W., Wach, C., Schnitzler, A. \& Südmeyer, M. 2012. Motor-cortical oscillations in early stages of Parkinson's disease. J Physiol 590, 3203-3012.

Pollok, B., Latz, D., Krause, V., Butz, M. \& Schnitzler, A. 2014. Changes of motorcortical oscillations associated with motor learning. Neuroscience 275, 47-53.

Robson, S., Brookes, M., Hall, E., Palaniyappan, L., Kumar, J., Skelton, M., Christodoulou, N., Qureshi, A., Jan, F., Katshu, M., Liddle, E., Liddle, P. \& Morris, P. 2016. Abnormal visuomotor processing in schizophrenia. NeuroImage Clin doi:10.1016/j.nicl.2015.08.005 
Salmelin, R. \& Hari, R. 1994. Spatiotemporal characteristics of sensorimotor neuromagnetic rhythms related to thumb movement. Neuroscience $60,537-550$.

Sarvas, J. 1987. Basic mathematical and electromagnetic concepts of the biomagnetic inverse problem. Phys Med Biol 32, 11-22.

Smith, J., Martin, P., Gandevia, S. \& Taylor, J. 2007. Sustained contraction at very low forces produces prominent supraspinal fatigue in human elbow flexor muscles. $J$ Appl Physiol 103, 560-568.

Solis-Escalante, T., Müller-Putz, G., Pfurtscheller, G. \& Neuper, C. 2012. Cue-induced beta rebound during withholding of overt and covert foot movement. Clin Neurophysiol 123, 1182-1190.

Steens, A., Heersema, D., Maurits, N., Renken, R. \& Zijdewind, I. 2012. Mechanisms underlying muscle fatigue differ between multiple sclerosis patients and controls: a combined electrophysiological and neuroimaging study. Neuroimage 59, 31103118.

Studer, B., Koeneke, S., Blum, J. \& Jäncke, L. 2010. The effects of practice distribution upon the regional oscillatory activity in visuomotor learning. Behav Brain Funct 6, 8.

Taylor, J., Gandevia, S., 2008. A comparison of central aspects of fatigue in submaximal and maximal voluntary contractions. J Appl Physiol 104, 542-550.

Taylor, J., Todd, G. \& Gandevia, S. 2006. Evidence for a supraspinal contribution to human muscle fatigue. Clin Exp Pharmacol Physiol 33, 400-405. 
Tecchio, F., Porcaro, C., Zappasodi, F., Pesenti, A., Ercolani, M. \& Rossini, P. 2006. Cortical short-term fatigue effects assessed via rhythmic brain-muscle coherence. Exp Brain Res 174, 144-151.

van Duinen, H., Renken, R., Maurits, N. \& Zijdewind, I. 2007. Effects of motor fatigue on human brain activity, an fMRI study. Neuroimage 35, 1438-1449.

Vrba, J. \& Robinson, S. 2001. Signal processing in magnetoencephalography. Methods $25,249-271$. 
Table 1. Actual wrist flexion force during the test contractions at a target force of $40 \% \mathrm{MVF}_{\mathrm{b}}$. 'Pre'/'Post': pre-/post-intervention. Steadiness: within participant standard deviation. Data are group means $\pm \operatorname{SEM}(n=11)$.

\begin{tabular}{lcccc}
\hline & \multicolumn{2}{c}{ Control Session } & \multicolumn{2}{c}{ Fatigue Session } \\
& Pre & Post & Pre & Post \\
& & & & \\
\hline Force $\left(\% \mathrm{MVF}_{\mathrm{b}}\right)$ & $40.00 \pm 0.17$ & $40.05 \pm 0.15$ & $39.87 \pm 0.15$ & $39.80 \pm 0.18$ \\
Steadiness $\left(\% \mathrm{MVF}_{\mathrm{b}}\right)$ & $0.95 \pm 0.06$ & $0.99 \pm 0.08$ & $1.01 \pm 0.08$ & $1.12 \pm 0.10$ \\
\hline
\end{tabular}

Table 2. Average MNI coordinates and the most likely cortical locations (according to the Oxford-Harvard brain atlas) of MRMD, MRBD and PMBR. Data are group means \pm SEM (beta, $\mathrm{n}=10$; mu and PMBR, $\mathrm{n}=9$ ).

\begin{tabular}{ccccc}
\hline & \multicolumn{3}{c}{ MNI coordinates } & Cortical Location \\
& $\mathbf{X}$ & $\mathbf{y}$ & $\mathbf{z}$ & \\
\hline MRMD & $-41.9 \pm 1.7$ & $-30.6 \pm 2.0$ & $54.0 \pm 1.4$ & postcentral gyrus \\
MRBD & $-37.7 \pm 0.8$ & $-24.2 \pm 1.8$ & $53.7 \pm 1.3$ & postcentral gyrus \\
PMBR & $-33.4 \pm 1.8$ & $-21.8 \pm 2.1$ & $58.1 \pm 1.8$ & precentral gyrus \\
\hline
\end{tabular}

Table 3. MRMD, MRBD and PMBR expressed as a percentage change relative to resting (baseline) amplitude. 'Pre'/'Post': pre-/post-intervention. 


\begin{tabular}{cccccc}
\hline $\begin{array}{c}\text { Relative Amplitude } \\
\text { Loss/Increase }\end{array}$ & \multicolumn{2}{c}{ Control Session } & Fatigue Session & point \\
(\% resting amplitude) & Pre & Post & Pre & Post & interaction \\
& & & & & (P value) \\
MRMD & $-32.2 \pm 1.4$ & $-28.7 \pm 1.2$ & $-30.4 \pm 1.7$ & $-33.2 \pm 1.7$ & 0.215 \\
MRBD & $-41.1 \pm 1.0$ & $-31.9 \pm 1.1$ & $-34.9 \pm 0.9$ & $-36.4 \pm 0.9$ & 0.005 \\
PMBR & $21.8 \pm 2.0$ & $23.4 \pm 2.1$ & $24.1 \pm 2.4$ & $36.3 \pm 3.0$ & 0.095 \\
\hline
\end{tabular}


Figure 1. Experiment overview: (a) A participant seated within the MEG scanner. (b) A forearm positioned within the isometric wrist-flexion dynamometer (white arrow denotes direction of isometric force application). (c) An example of a target force profile with the real-time visual feedback of the performed contraction force overlaid. (d) A schematic of the experimental protocol. The intervention involved 40 contractions at either $5 \% \mathrm{MVF}_{\mathrm{b}}$ (control intervention) or $55 \% \mathrm{MVF}_{\mathrm{b}}$ (fatigue intervention; illustrated in the schematic). The same 14 test contractions were repeated pre- and post-intervention. (e) The data measurement periods (blue annotations) during and after the $40 \% \mathrm{MVF}_{\mathrm{b}}$ test contractions.

Figure 2. Maximum voluntary force (as an index of physical fatigue) at four time-points throughout the experimental sessions. $¥=$ significant difference between sessions $(\mathrm{P}<0.001$ following Bonferroni corrections). Data are group means $\pm \operatorname{SEM}(\mathrm{n}=11)$.

Figure 3. (a) Locations of peak MRMD during the $40 \% \mathrm{MVF}_{\mathrm{b}}$ test contractions for each individual participant (blue dots). (b) Absolute mu amplitude at location of peak MRMD during baseline period (20-25 s following contraction onset). (c) Timefrequency spectrograms extracted from locations of peak MRMD, showing task-related change from baseline oscillatory amplitude. (d) Average MRMD (1.5-6.5 s following contraction onset). Data are group means $\pm \operatorname{SEM}(n=9)$. 
Figure 4. (a) Locations of peak MRBD during the $40 \% \mathrm{MVF}_{\mathrm{b}}$ test contractions for each individual participant (blue dots). (b) Absolute beta amplitude at location of peak MRBD during baseline period (20-25 s following contraction onset). (c) Timefrequency spectrograms extracted from locations of peak MRBD, showing task-related change from baseline oscillatory amplitude. (d) Average MRBD (1.5-6.5 s following contraction onset). $*$ Significant session $\times$ time-point interaction $(\mathrm{P}<0.05)$. Data are group means \pm SEM $(\mathrm{n}=10)$.

Figure 5. (a) Locations of peak PMBR following the $40 \% \mathrm{MVF}_{\mathrm{b}}$ test contractions for each individual participant (blue dots). (b) Absolute beta amplitude at location of peak PMBR during baseline period (20-25 s following contraction onset). (c) Time-frequency spectrograms extracted from locations of peak PMBR, showing task-related change from baseline oscillatory amplitude. (d) Total PMBR amplitude (0-10 s following contraction offset). ${ }^{*}=$ Significant session $\times$ time-point interaction $(\mathrm{P}<0.05)$. Data are group means \pm SEM $(n=9)$. 

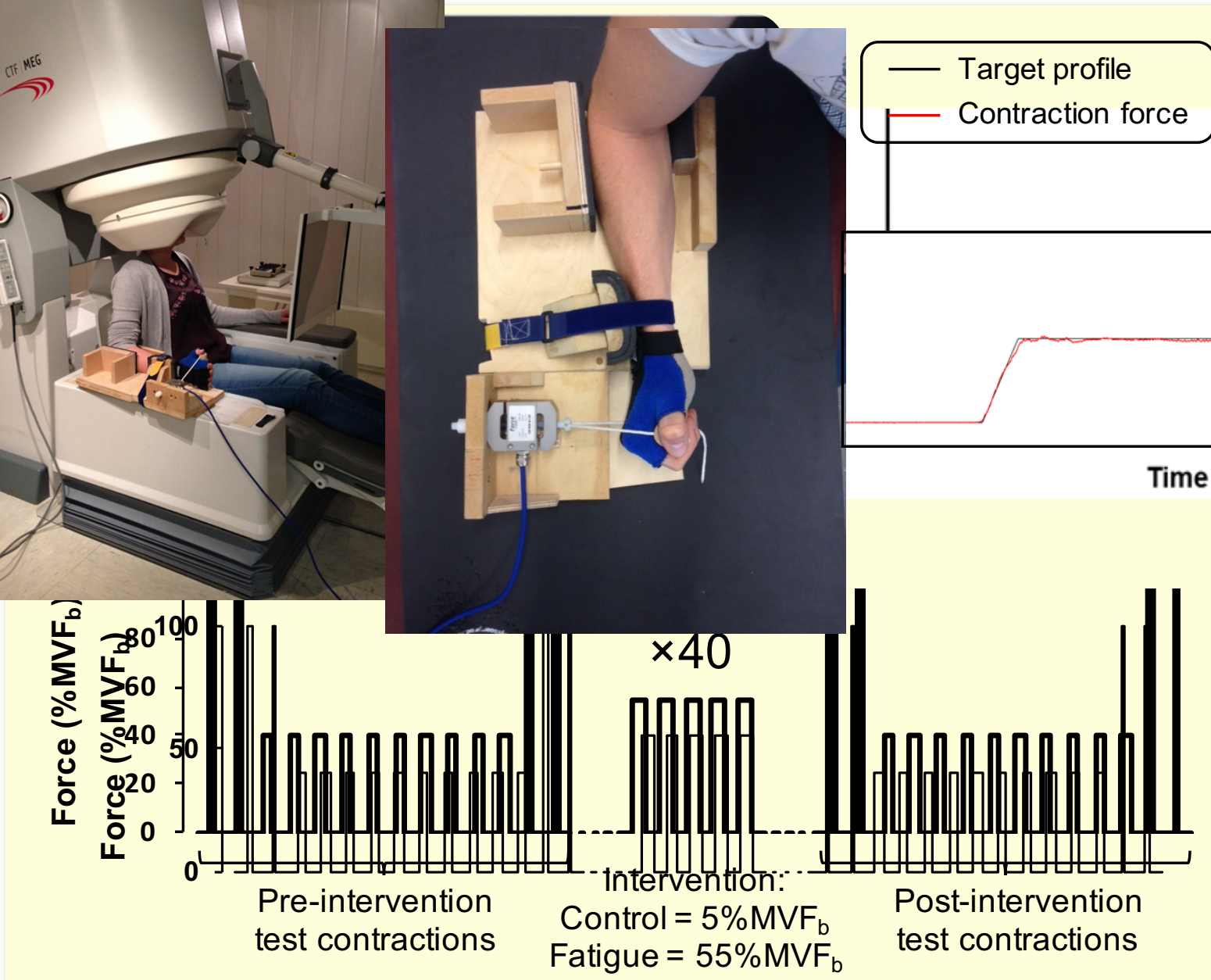

Test contractions:

$2 \times 100 \% M_{\mathrm{MVF}}$ : $\mathrm{MVF}_{\text {start }}$ - used to monitor physical fatigue $10 \times 40 \% \mathrm{MVF}_{\mathrm{b}}$ : used for measurement of MRMD, MRBD and PMBR (see below) $2 \times 100 \% \mathrm{MVF}_{\mathrm{b}}$ : $\mathrm{MVF}_{\text {end }}$ - used to monitor physical fatigue

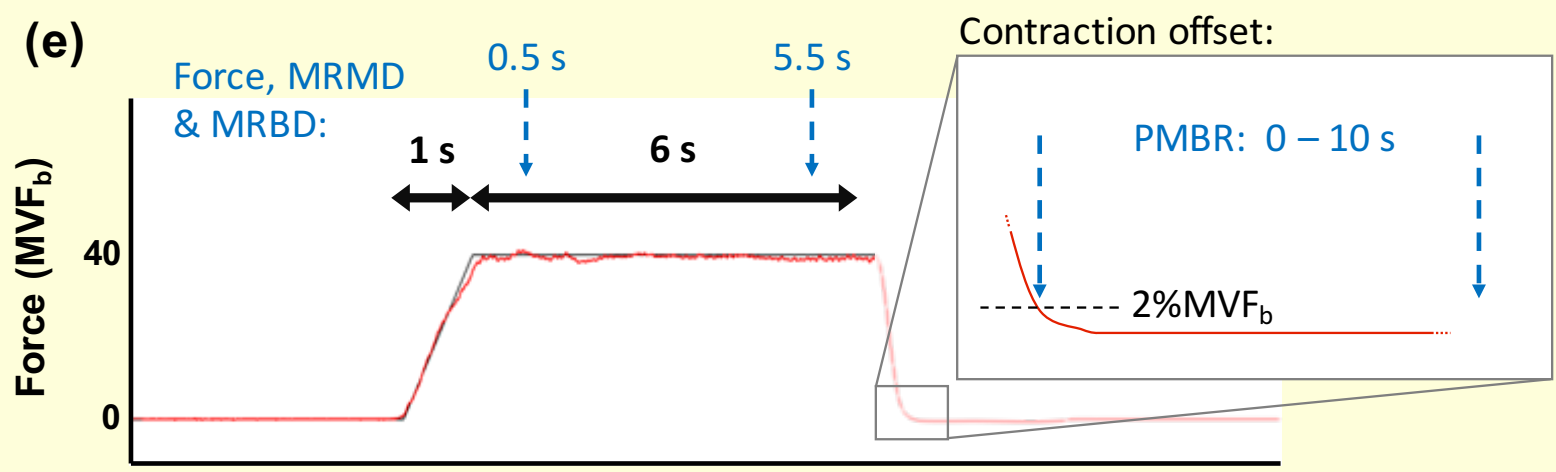


(a)

(b) $\quad$ CControl $\quad$ Fatigue

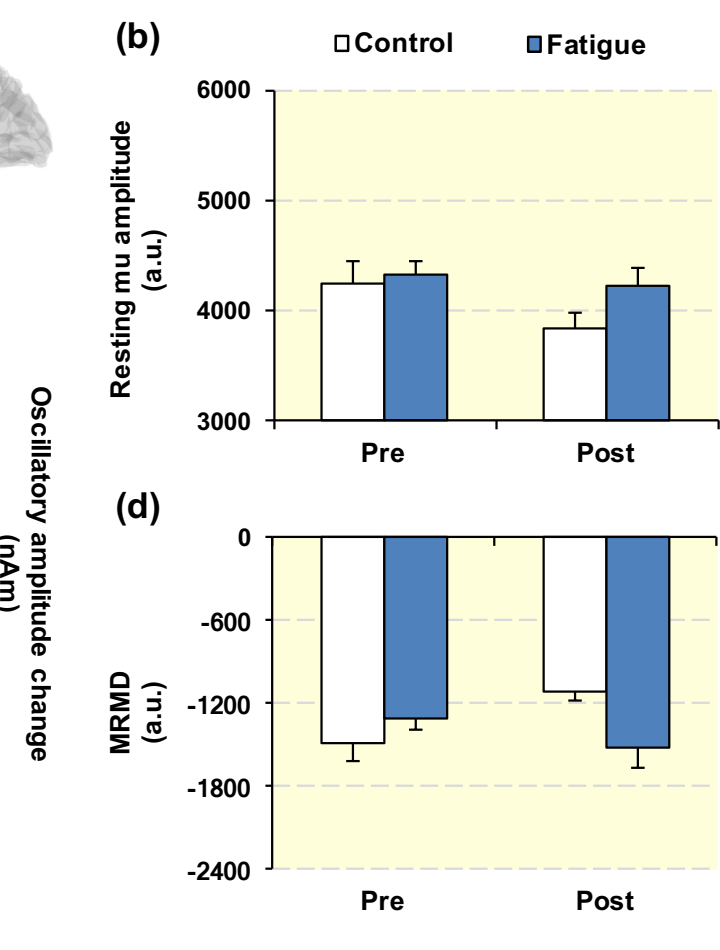

(c)

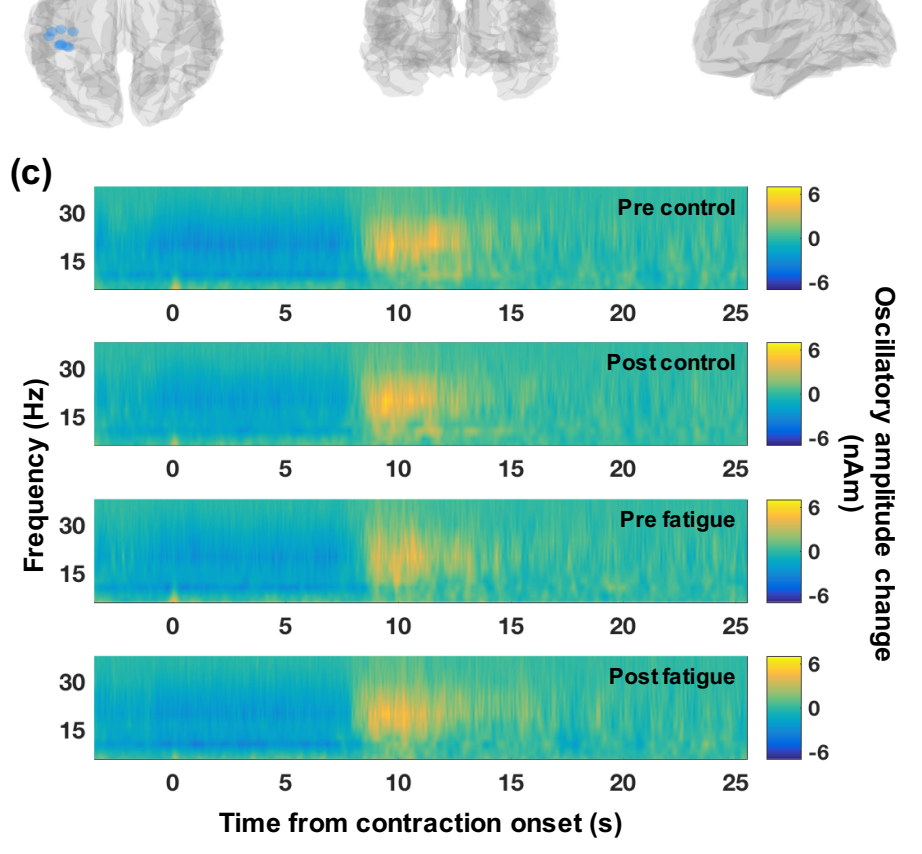


(a)

(b)

(c)

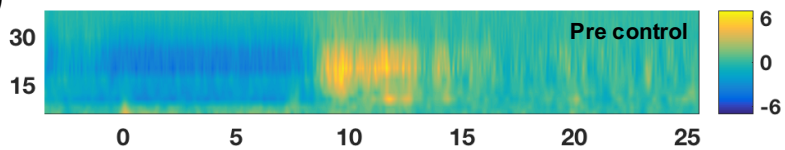

옹

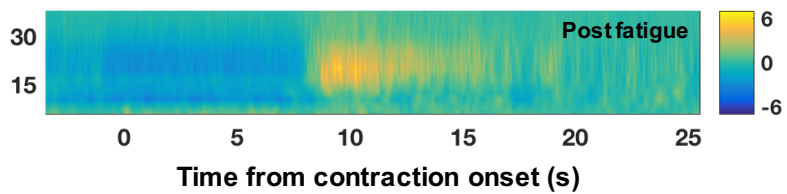

口Control

口Fatigue

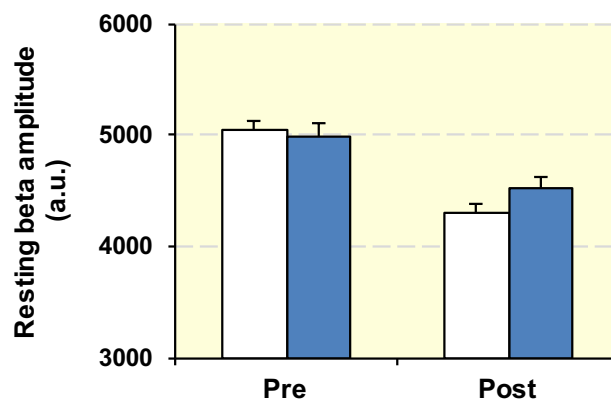

(d)

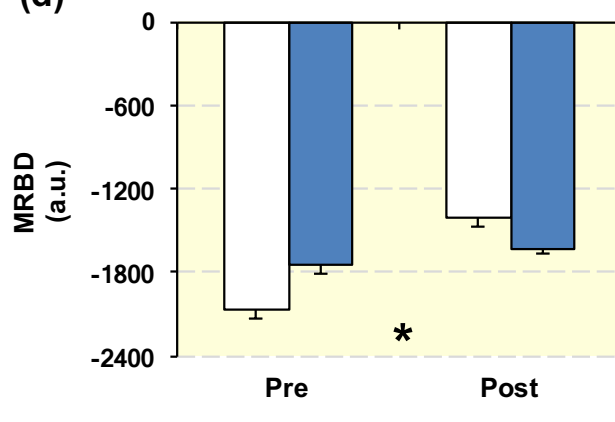


(a)

(b)

(c)

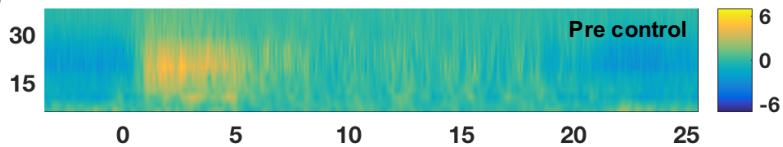

0

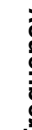

นั

온
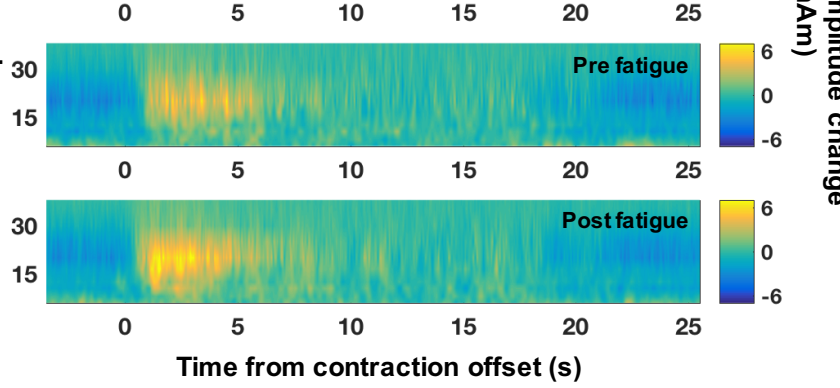

口Control aFatigue

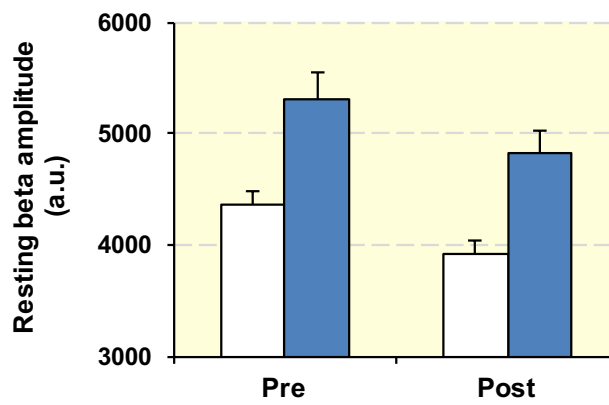

(d)

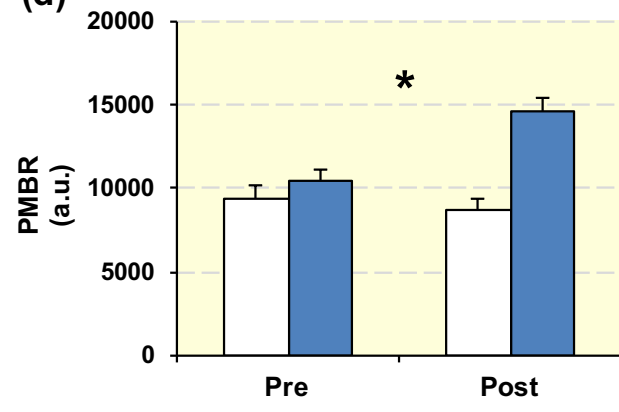

